\title{
The How and Why of Smart Features
}

\author{
R. Anderhalt, T. Nylese \\ EDAX Inc., 91 McKee Drive, Mahwah, NJ, 07430
}

For many years the manufacturers of all forms of analytical instrumentation have sought to make these systems easy to use. Much of the effort was to re-organize the parameters into the sequence of steps that a user would be most likely to use. Still, many of the parameters were variable and the optimal configuration of these parameters would require a certain amount of knowledge or expertise. To help the user with less experience, the next logical step in the evolution of analytical instrumentation was to develop smart features. The goal was that the variable parameters might be auto-configured in a way that was comparable to what the experienced user would have chosen.

In the case of energy-dispersive X-ray spectroscopy (EDS) an approach has been tried for the auto pulse processing time where the process time would be automatically adjusted for the X-ray count rate so that the dead time would stay within a reasonable range. In terms of what a knowledgeable user might have done, this might have replicated what their choice would have been with the exception that oftentimes the expert would actually have lowered the count rate so that the EDS detector resolution would have been at its best. In other cases, rather than accepting the count rate as it was, it might have been increased for an X-ray map. A smart feature should allow that it can be turned off or disabled, at least temporarily.

In many cases the parameters and variables of an EDS system can be optimized and there are many frequently asked questions by users about what beam voltage, working distance, count rate, dead time, map resolution, map quality, image resolution, how to correct image drift during relatively long procedures, or how to capture an image that is not too noisy or that does not take too much time. Many experienced EDS analysts know that the answer to almost all of these questions is that "it depends". In some cases it depends on some other parameter and we may very well have the statistical relationship of a dependent variable which depends on some independent variable. We will address the last question in a little detail.

Determining the frame time for image collection is clearly an example in which the image collect time should depend on how much noise is in our image. If the image is judged to be too noisy, the time to collect the image should be increased (fig. 1). This appears to be an iterative approach and not all that satisfactory to an operator (Fig. 2). If we have some way to measure the noise in the image it should be possible to calculate the time needed to collect a good image that did not take too long to collect. It would also be possible to collect the image in one iteration without having to re-do the collection. The noise in the image can be measured by the standard deviation or variance of replicate samplings of a single pixel or small region of the image and the time adjusted accordingly (Fig. 2). Many of the parameters that we must configure depend on a single additional variable which is known and in some cases it depends primarily on one variable with a lesser dependence on another. The approach for image collection would seem to be an example with a single independent variable but there could also be another variable such as a tendency of the sample to charge if we dwell too long at a given point. In this example we are justified in assuming that the sample is conductive or, if not, that it should be coated with a conductor. 

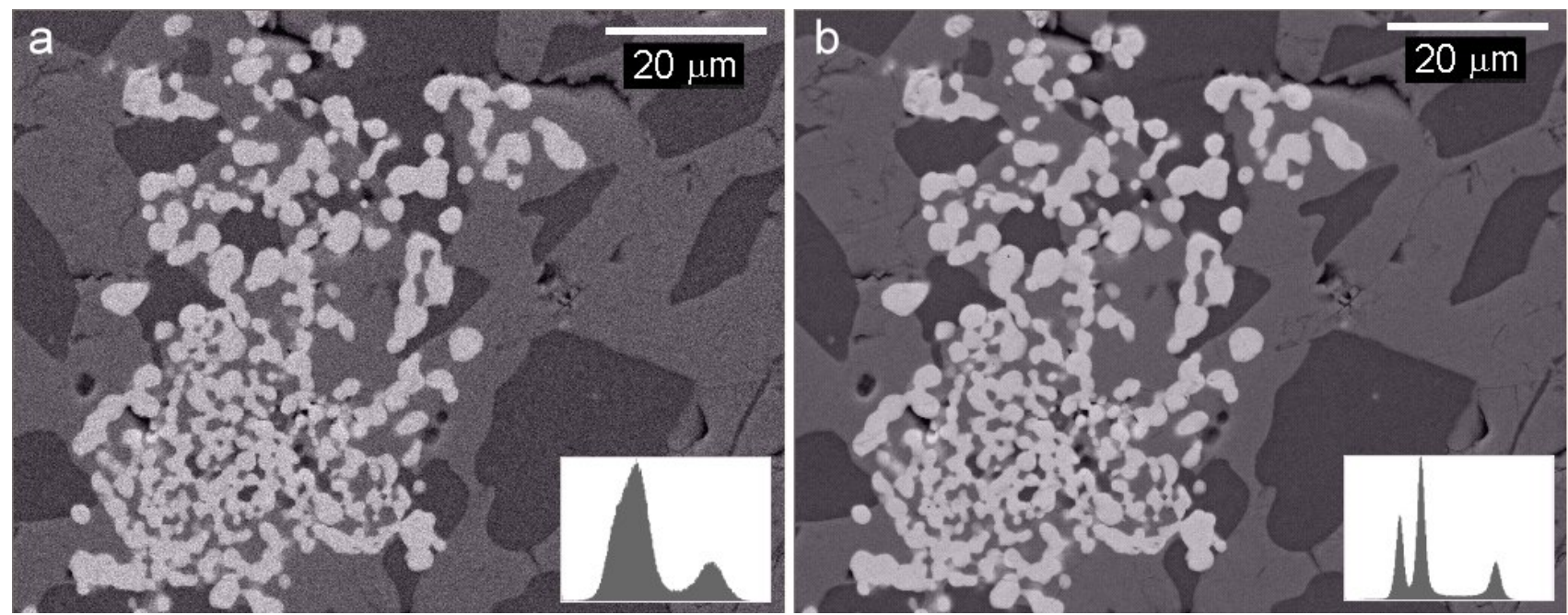

Fig. 1.Two images are shown with the image histogram shown as an insert at the lower right. 1a) this image has more noise and even though the human eye is able to resolve the 3 phases, the image histogram does not resolve the two darker phases. 1b) has an image that took more time to collect and provides an unambiguous resolution of the 3 phases as can be seen in the histogram.
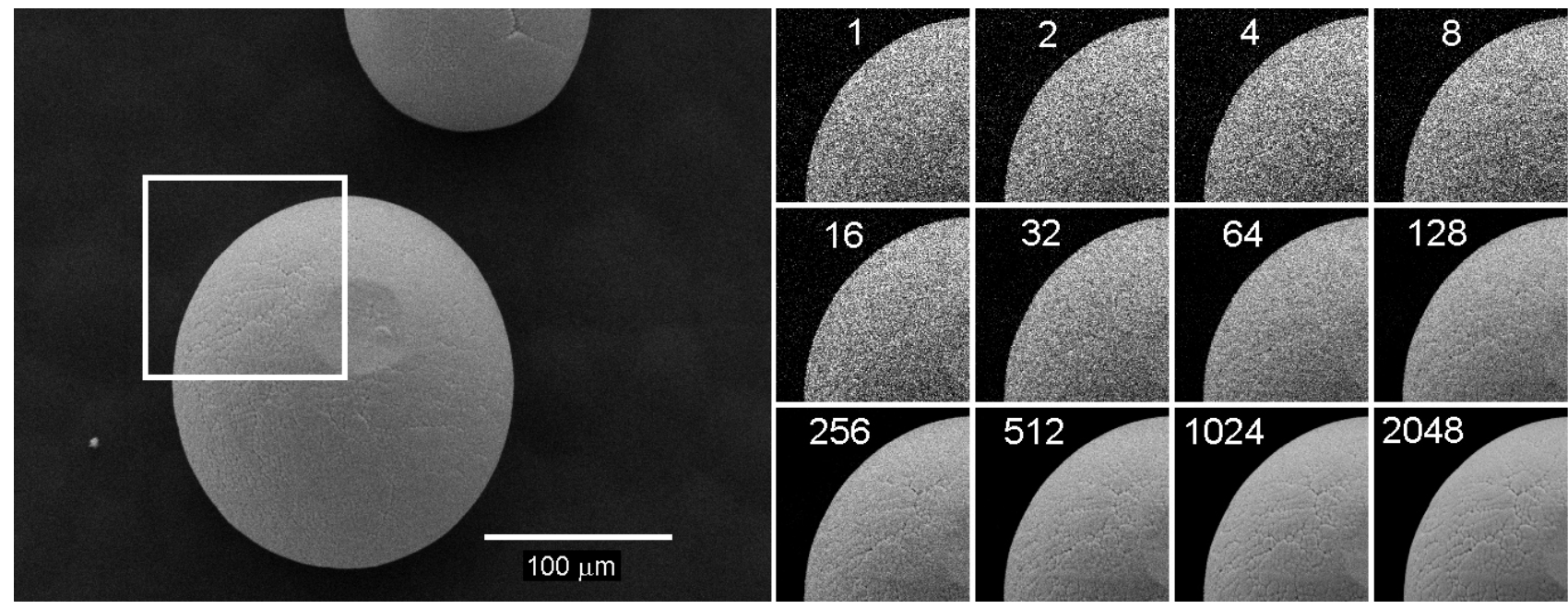

Fig. 2. Images were collected of the spherical particle shown at the left using a secondary electron detector with a beam voltage of $20 \mathrm{kV}$. The length of time was increased to collect multiple images using a parameter called "reads" which is a multiplier of the dwell time at each pixel beyond the initial read. The image quality improves with a longer acquisition time, however, there is some point where it would generally be determined that the additional time was not worth the slight improvement in image quality. These images were collected with a low beam current $(0.03 \mathrm{nA})$-much lower than could be justified for this relatively low magnification. The image collected for 2048 reads at 1024x800 pixel resolution took almost 3 minutes to collect. The smart feature for optimizing the image collection made the determination that about 400 reads would be adequate in this case or about a 40 second frame time. 